\title{
ON THE THEOREM OF FEJER-RIESZ
}

\author{
A. ZYGMUND
}

1. Statement of results. Let

$$
f(z)=a_{0}+a_{1} z+a_{2} z^{2}+\cdots+a_{n} z^{n}+\cdots
$$

be a function regular for $|z| \leqq 1$. The well known inequality of Fejér and Riesz asserts that

$$
\int_{D}|f(z)||d z| \leqq \frac{1}{2} \int_{C}|f(z)||d z|,
$$

where $C$ is the circumference $|z|=1$, and $D$ any of its diameters. ${ }^{1}$

For $f(z)=F^{\prime}(z)$, the inequality (2) takes the form

$$
\int_{D}\left|F^{\prime}(z)\right||d z| \leqq \frac{1}{2} \int_{C}\left|F^{\prime}(z)\right||d z|
$$

which shows that the total variation of $F(z)$ along $D$ does not exceed half of the total variation of $F$ along $C$. In this form the inequality remains valid for harmonic functions. Let $z=\rho e^{i \theta}$. If $U(z)=U(\rho, \theta)$ is harmonic for $|z| \leqq 1$, the total variation of $F$ along $D$ does not exceed half of the total variation of $F$ along $C . .^{2}$ In symbols,

$$
\int_{D}\left|U_{\rho}\right| d \rho \leqq \frac{1}{2} \int_{C}\left|U_{\theta}\right| d \theta .
$$

Let $V(z)=V(\rho, \theta)$ be the harmonic function conjugate to $U$. In (4) we may replace $U_{\rho}$ by $\rho^{-1} V_{\theta}$. Writing $U_{\theta}=u, V_{\theta}=v$, we obtain an equivalent form of the inequality (4), namely

$$
\int_{D}\left|\frac{v(z)}{z}\right||d z| \leqq \frac{1}{2} \int_{C}|u(z)||d z| .
$$
1945.

Received by the editors October 22, 1945, and, in revised form, November 19,

${ }^{1}$ L. Fejér and F. Riesz, Ueber eine funktionentheoretische Ungleichung, Math. Zeit. vol. 11 (1921) pp. 305-314.

${ }_{2}$ The inequality (4), with $1 / 2$ on the right replaced by an undetermined constant $A$, was first proved by B. N. Prasad, On the summability of power series and the bounded variation of power series, Proc. London Math. Soc. vol. 35 (1933) pp. 407-424. That $C=1 / 2$ was shown in F. Riesz, Eine Ungleichung für harmonische Funktionen, Monatshefte für Mathematik und Physik vol. 43 (1936) pp. 401-406, and A. Zygmund, Some points in the theory of trigonometric and power series, Trans. Amer. Math. Soc. vol. 36 (1934) pp. 586-617, especially p. 599. 
It is valid for any pair of functions

$$
\begin{aligned}
& u(z)=u(\rho, \theta)=\frac{a_{0}}{2}+\sum_{\nu=1}^{\infty}\left(a_{\nu} \cos \nu \theta+b_{\nu} \sin \nu \theta\right) \rho^{\nu}, \\
& v(z)=v(\rho, \theta)=\sum_{\nu=1}^{\infty}\left(a_{\nu} \sin \nu \theta-b_{\nu} \cos \nu \theta\right) \rho^{\nu},
\end{aligned}
$$

harmonic and conjugate in $|z| \leqq 1$, provided that $v(0)=0$.

The purpose of this note is to prove the following complement to (4).

THEOREM 1. Let

$$
U(z)=\frac{A_{0}}{2}+\sum_{\nu=1}^{\infty}\left(A_{\nu} \cos \nu \theta+B_{\nu} \sin \nu \theta\right) \rho^{\nu} \quad\left(z=\rho e^{i \theta}\right)
$$

be a function harmonic for $|z| \leqq 1$, and let $U_{n}(z)$ be the nth partial sum of the series (8). For every $n$, the total variation of $U_{n}(z)$ over $D$ does not exceed $2 / \pi$ times the total variation of $U(z)$ over $C$. The constant $2 / \pi$ here cannot be replaced by any smaller number.

We shall prove this result in the following equivalent form.

THEOREM 1'. Let $u(z)$ and $v(z)$, given by (6) and (7), be harmonic for $|z| \leqq 1$ and conjugate (in particular, $v(0)=0$ ). Let $v_{n}(z)$ be the nth partial sum of the series (7). Then

$$
\int_{D}\left|\frac{v_{n}(z)}{z}\right||d z| \leqq \frac{2}{\pi} \int_{C}|u(z)||d z|
$$

for all $n$, the factor $2 / \pi$ on the right being the best possible.

Taking $u(z)=z f(z)$, so that $v(z)=-i z f(z)$, we deduce from (9) the following corollary.

THEOREM 2. Let $f(z)$ be a function regular for $|z| \leqq 1$ and let $s_{n}(z)$ be the nth partial sum of the series (1). Then for all $n$

$$
\int_{D}\left|s_{n}(z)\right||d z| \leqq \frac{2}{\pi} \int_{C}|f(z)||d z| \text {. }
$$

In all these results the assumption that the functions are harmonic (or regular) inside and on $C$ can obviously be relaxed, and more general results may be obtained from the special ones by routine passages to the limit. ${ }^{3}$ Thus, for example, in Theorem 2 we may assume

See also $\$ 3$, below. 
that $f(z)$ is regular for $|z|<1$ and continuous for $|z| \leqq 1$. The partial sums $s_{n}(z)$ for such an $f$ may be unbounded, and it is of interest to note that in the passage from (2) to (10) the increase of the coefficient on the right (from $1 / 2$ to $2 / \pi=0.64 \cdots$ ) is not significant.

That $2 / \pi$ is the best constant in Theorem $1^{\prime}$ (and so in Theorem 1) is easily seen from the example

$$
u(z)=1 / 2+\rho R \cos \theta+\rho^{2} R^{2} \cos 2 \theta+\cdots \quad\left(z=\rho e^{i \theta}\right)
$$

where $R$ is a fixed positive number less than 1. The integral on the right of (9) is then $\pi$. Taking $n=1$ and the segment $(-i, i)$ for $D$ we find for the integral on the left the value $2 R$. Since $R$ may be as close to 1 as we wish, the conclusion follows.

As we shall see later, for each $n>1$ the constant $2 / \pi$ on the right can be replaced by a constant $C_{n}<2 / \pi$, and clearly $2 / \pi=C_{1} \geqq C_{2}$ $\geqq \cdots \geqq C_{n} \geqq \cdots$. One might expect that $C_{n} \rightarrow 1 / 2$, in accordance with (5). It is however not so, and the difference $C_{n}-1 / 2$ stays above a positive number. This fact has close connection with the Gibbs' phenomenon.

2. Proof of Theorem $1^{\prime}$. We shall use the formula

$$
v_{n}(\rho, \theta)=-\frac{1}{\pi} \int_{-\pi}^{\pi} u(1, \theta+t) \rho \Delta_{n}(\rho, t) d t
$$

where

$$
\Delta_{n}(\rho, t)=\sum_{\nu=1}^{n} \rho^{\nu-1} \sin \nu t .
$$

Taking, as we may, for $D$ the segment $(-1,+1)$ of the real axis, we get

$$
\int_{D}\left|\frac{v_{n}(z)}{z}\right||d z| \leqq \frac{1}{\pi} \int_{-\pi}^{\pi}|u(1, t)|\left\{\int_{-1}^{+1}\left|\Delta_{n}(\rho, t)\right| d \rho\right\} d t,
$$

and our problem reduces to showing that

$$
\int_{-1}^{+1}\left|\Delta_{n}(\rho, t)\right| d \rho \leqq 2, \quad \text { for } n=1,2, \cdots,-\pi \leqq t \leqq \pi .
$$

That the integral on the left here is bounded as a function of $n$ would be a simple matter to show. Complications arise when we want to show that the integral in question does not exceed 2.

We shall need the following lemma. 
LEMma. Let $0 \leqq t \leqq \pi$. For every $\mu$ positive and odd, the values of the trigonometric polynomials

$$
\begin{gathered}
\sin t / 1+\sin 3 t / 3+\cdots+\sin \mu t / \mu, \\
\sin t / 1+\sin 3 t / 3+\cdots+\sin (\mu-2) t /(\mu-2)+\sin \mu t / 2 \mu
\end{gathered}
$$

are contained between 0 and 1 .

Taking temporarily this lemma for granted, we shall proceed with the proof of the inequality (13). Let us consider the auxiliary function

$$
\Delta_{n}^{*}(\rho, t)=\sum_{\nu=1}^{n} \rho^{\nu-1} \sin \nu t=\sum_{\nu=1}^{n-1} \rho^{\nu-1} \sin \nu t+\frac{\rho^{n-1} \sin n t}{2} .
$$

We easily find that

$$
\Delta_{n}^{*}(\rho, t)=\frac{\frac{\sin t}{2}\left[2\left(1-\rho^{n} \cos n t\right)-\rho^{n-1} \frac{\sin n t}{\sin t}\left(1-\rho^{2}\right)\right]}{1-2 \rho \cos t+\rho^{2}} .
$$

We note that, for $0 \leqq \rho \leqq 1$,

$$
\begin{aligned}
2\left(1-\rho^{n} \cos n t\right)- & \rho^{n-1} \frac{\sin n t}{\sin t}\left(1-\rho^{2}\right) \geqq 2\left(1-\rho^{n}\right)-n \rho^{n-1}\left(1-\rho^{2}\right) \\
& =(1-\rho)\left[2\left(1+\rho+\cdots+\rho^{n-1}\right)-\rho^{n-1} n(1+\rho)\right] \\
& \geqq(1-\rho)\left(2 n \rho^{n-1}-2 n \rho^{n-1}\right)=0 .
\end{aligned}
$$

Thus $\Delta_{n}^{*}$ (unlike $\Delta_{n}$ ) is non-negative for $0 \leqq t \leqq \pi$, and so nonpositive in the interval $(-\pi, 0)$. This remark applies, in particular, to the trigonometric polynomial

$$
\Delta_{n}^{*}(1, t)=\sum_{\nu=1}^{n} \sin \nu t
$$

Let us now fix $t, 0 \leqq t \leqq \pi$. In the proof of (13) we shall consider various special cases.

Case (1). $\Delta_{n}(\rho, t) \geqq 0, \Delta_{n}(\rho, t+\pi) \leqq 0$ for $0 \leqq \rho \leqq 1$.

Thus

$$
\begin{aligned}
\int_{-1}^{+1}\left|\Delta_{n}(\rho, t)\right| d \rho & =\int_{0}^{1}\left[\Delta_{n}(\rho, t)-\Delta_{n}(\rho, t+\pi)\right] d \rho \\
& =\int_{0}^{1}\left\{\sum_{\nu \leqq n} \rho^{\nu-1} \sin \nu t-\sum_{\nu \leq n}(-1)^{\nu} \rho^{\nu-1} \sin \nu t\right\} d \rho \\
& =2 \sum_{\nu \leqq n, \nu \text { odd }} \frac{\sin \nu t}{\nu} \leqq 2,
\end{aligned}
$$


by the lemma.

Case (ii). $\Delta_{n}\left(\rho_{0}, t\right)<0$, for some $\rho_{0}, 0 \leqq \rho_{0} \leqq 1$. We may assume that $0<\rho_{0}<1$.

This cannot happen for $n=1$. Thus $n \geqq 2$. We shall show that in this case, for all $\rho, 0 \leqq \rho \leqq 1$ :

(a) $\Delta_{n}(\rho, t+\pi)<0$.

(b) $\left|\Delta_{n}(\rho, t)\right|$ is majorized both by $\Delta_{n-1}(\rho, t)$ and $\Delta_{n+1}(\rho, t)$ (in particular, the latter quantities must be non-negative).

For suppose that

$$
\Delta_{n}(\rho, t)=\sin t+\rho_{0} \sin 2 t+\cdots+\rho_{0}^{n-1} \sin n t<0 .
$$

We know that for all $\rho, 0 \leqq \rho \leqq 1$,

$$
\begin{aligned}
\Delta_{n}^{*}(\rho, t)= & \sin t+\rho \sin 2 t+\cdots \\
& +\rho^{n-2} \sin (n-1) t+2^{-1} \rho^{n-1} \sin n t \geqq 0, \\
\Delta_{n+1}^{*}(\rho, t)= & \sin t+\rho \sin 2 t+\cdots \\
& +\rho^{n-1} \sin n t+2^{-1} \rho^{n} \sin (n+1) t \geqq 0 .
\end{aligned}
$$

A comparison of these inequalities shows that

$$
\sin n t<0, \quad \sin (n+1) t>0 .
$$

Thus, since $\Delta_{n}=\Delta_{n}^{*}+2^{-1} \rho^{n-1} \sin n t$, we have

$$
\begin{aligned}
2^{-1} \rho^{n-1} \sin n t & \leqq \Delta_{n}(\rho, t) \leqq \Delta_{n}^{*}(\rho, t), \\
\left|\Delta_{n}(\rho, t)\right| & \leqq\left|\Delta_{n}^{*}(\rho, t)\right|+\left|2^{-1} \rho^{n-1} \sin n t\right| \\
& =\Delta_{n}^{*}(\rho, t)-2^{-1} \rho^{n-1} \sin n t=\Delta_{n-1}(\rho, t) .
\end{aligned}
$$

Similarly, the formula $\Delta_{n}=\Delta_{n+1}^{*}-2^{-1} \rho^{n} \sin (n+1) t$ and (15) imply

$$
\begin{aligned}
-2^{-1} \rho^{n} \sin (n+1) t & \leqq \Delta_{n}(\rho, t) \leqq \Delta_{n+1}^{*}(\rho, t), \\
\left|\Delta_{n}(\rho, t)\right| & \leqq\left|\Delta_{n}^{*}+1(\rho, t)\right|+\left|2^{-1} \rho^{n} \sin (n+1) t\right| \\
& =\Delta_{n+1}^{*}(\rho, t)+2^{-1} \rho^{n} \sin (n+1) t=\Delta_{n+1}(\rho, t) .
\end{aligned}
$$

Thus assertion (b) is proved. To prove (a) we replace $t$ by $t+\pi$ in the equations

$$
\begin{aligned}
& \Delta_{n}(\rho, t)=\Delta_{n}^{*}(\rho, t)+2^{-1} \rho^{n-1} \sin n t, \\
& \Delta_{n}(\rho, t)=\Delta_{n+1}^{*}(\rho, t)-2^{-1} \rho^{n} \sin (n+1) t .
\end{aligned}
$$

If $n$ is even, the first of these resulting equations, coupled with the inequalities $\Delta_{n}^{*}(\rho, t+\pi) \leqq 0, \sin n t<0$, shows that $\Delta_{n}(\rho, t+\pi)<0$. If $n$ is odd, we similarly argue with the second equation. Thus (a) is proved.

Using (a) and (b), we see that in case (ii) 


$$
\begin{aligned}
\int_{-1}^{+1}\left|\Delta_{n}(\rho, t)\right| d \rho & \leqq \int_{0}^{1}\left\{\Delta_{n \pm 1}(\rho, t)-\Delta_{n}(\rho, t+\pi)\right\} d \rho \\
& =\int_{0}^{1}\left\{\sum_{\nu=1}^{n \pm 1} \rho^{\nu-1} \sin \nu t-\sum_{\nu=1}^{n}(-1)^{\nu} \rho^{\nu-1} \sin \nu t\right\} d \rho .
\end{aligned}
$$

If $n$ is even, we take the sign + in $n \pm 1$, and the last integral becomes

$$
2\left\{\sum_{\nu \leqq n-1, \nu \text { odd }} \frac{\sin \nu t}{\nu}+\frac{1}{2} \frac{\sin (n+1) t}{n+1}\right\} \leqq 2,
$$

by the lemma. If $n$ is odd ( $n \geqq 3$ ), we take the sign - in $n \pm 1$, and the integral in question takes the form

$$
2\left(\sum_{\nu \leqq n-2, \nu \text { odd }} \frac{\sin \nu t}{\nu}+\frac{1}{2} \frac{\sin n t}{n}\right) \leqq 2,
$$

again by the lemma. Thus (13) holds in case (ii).

Case (iii). $\Delta_{n}\left(\rho_{0}, t+\pi\right)>0$ for some $\rho_{0}, 0 \leqq \rho_{0} \leqq 1$.

To prove that (13) holds in this case, we observe that, since $\Delta_{n}(\rho, t)$ is odd in $t$,

$$
\Delta_{n}\left(\rho_{0}, t+\pi\right)=-\Delta_{n}\left(\rho_{0}, \pi-t\right)=-\Delta_{n}\left(\rho_{0}, t^{\prime}\right),
$$

where $t^{\prime}=\pi-t$. Thus $0 \leqq t^{\prime} \leqq \pi, \Delta_{n}\left(\rho_{0}, t^{\prime}\right)$ is negative, and we are in case (ii). It follows that (13) holds if we replace there $t$ by $t^{\prime}$. But

$$
\begin{aligned}
\int_{-1}^{+1}\left|\Delta_{n}(\rho, t)\right| d \rho & =\int_{-1}^{+1}\left|\Delta_{n}(\rho, t-\pi)\right| d \rho \\
& =\int_{-1}^{+1}\left|\Delta_{n}\left(\rho, t^{\prime}\right)\right| d \rho \leqq 2,
\end{aligned}
$$

and (13) holds again.

Cases (i), (ii), (iii) exhaust all possibilities, since the simultaneous occurrence of the inequalities $\Delta_{n}\left(\rho_{0}, t\right)<0, \Delta_{n}\left(\rho_{1}, t+\pi\right)>0$ is excluded by case (ii). Thus for the completion of the proof of Theorem $1^{\prime}$ we need only prove the lemma.

In estimating the polynomial

$$
\begin{aligned}
\frac{\sin t}{1}+\frac{\sin 3 t}{3}+ & \cdots+\frac{\sin \mu t}{\mu} \\
& =\int_{0}^{t}(\cos u+\cos 3 u+\cdots+\cos \mu u) d u \\
& =\int_{0}^{t} \frac{\sin (\mu+1) u}{2 \sin u} d u
\end{aligned}
$$


we may assume that $0 \leqq t \leqq \pi / 2$, since replacing $t$ by $\pi-t$ does not change the value of the polynomial. It is clear that the maximum of the last integral is attained for $t=\pi /(\mu+1)$, and is equal to

$$
\int_{0}^{\pi /(\mu+1)} \frac{\sin (\mu+1) u}{2 \sin u} d u=\int_{0}^{\pi} \frac{\sin u}{2(\mu+1) \sin (u / \mu+1)} d u .
$$

For fixed $u, 0 \leqq u \leqq \pi$, and $\mu \geqq 1$, the minimum of the last denominator is attained when $\mu=1$. For this particular value of $\mu$ the last integral becomes $2^{-1} \int_{0}^{x} \cos (u / 2) d u=1$, and one-half of the lemma is proved.

Similarly, assuming, as we may, that $\mu \geqq 3$, we get

$$
\begin{aligned}
\sin t+\frac{\sin 3 t}{3}+\cdots+\frac{\sin \mu t}{2 \mu} & =\int_{0}^{t} \frac{\sin \mu u}{2 \tan u} d u \leqq \int_{0}^{\pi / \mu} \frac{\sin \mu u}{2 \tan u} d u \\
& \leqq \int_{0}^{\pi / \mu} \frac{\sin \mu u}{2 \sin u} d u \leqq 1
\end{aligned}
$$

by the result just obtained. This completes the proof of the lemma.4

3. Additional remarks. (i) Let $C_{n}$ be the least number such that

$$
\int_{D}\left|\frac{v_{n}(z)}{z}\right||d z| \leqq C_{n} \int_{C}|u(z)||d z|
$$

for all functions $u(z)$ harmonic in $|z| \leqq 1$. We know that $C_{1}=2 / \pi$, and the argument just completed clearly shows that $C_{n}<2 / \pi$ for $n=2,3, \ldots$. The numbers $C_{1} \geqq C_{2} \geqq C_{3} \geqq \ldots$ tend to a limit $C^{*} \geqq 1 / 2$. Combining the estimates in cases (i), (ii), (iii) with the inequalities of the lemma, we find that

$$
\begin{aligned}
C^{*} \leqq \lim _{m \rightarrow \infty} \frac{1}{\pi} \int_{0}^{\pi / m} \frac{\sin m u}{\sin u} d u & =\lim _{m \rightarrow \infty} \frac{1}{\pi} \int_{0}^{\pi / m} \frac{\sin m u}{u} d u \\
& =\frac{1}{\pi} \int_{0}^{\pi} \frac{\sin u}{u} d u .
\end{aligned}
$$

On the other hand, let $R$ be any positive number less than 1 and let $u(z)=u_{R}(z)=\pi^{-1}\left(1 / 2+R \rho \cos \theta+R^{2} \rho^{2} \cos 2 \theta+\cdots\right), \quad z=\rho e^{i \theta}$, so that $\int_{C}|u(z)||d z|=1$. If $t, 0<t<\pi$, is the angle of $D$ with the positive real axis, then

\footnotetext{
- The author is indebted to a referee for pointing out a slip in the initial proof of the lemma.
} 


$$
\begin{aligned}
\int_{D}\left|\frac{v_{n}(z)}{z}\right||d z| & =\int_{-1}^{+1}\left|\Delta_{n}(\rho, t)\right| d \rho \\
& \geqq \int_{0}^{+1}\left\{\Delta_{n}(\rho, t)-\Delta_{n}(\rho, t+\pi)\right\} d \rho \\
& =2\left\{R \frac{\sin t}{1}+R^{3} \frac{\sin 3 t}{3}+\cdots+R^{\mu} \frac{\sin \mu t}{\mu}\right\},
\end{aligned}
$$

where $\mu$ is the largest odd integer not greater than $n$. Taking $R$ as close to 1 as we wish, we see that the last sum comes arbitrarily close to

$$
2\left(\frac{\sin t}{1}+\frac{\sin 3 t}{3}+\cdots+\frac{\sin \mu t}{\mu}\right)
$$

which is a partial sum of the Fourier series of the function $(\pi / 2) \operatorname{sign} t$ $(-\pi<t<\pi)$. This function has a jump at $t=0$ so that the partial sums (22) must display Gibbs' phenomenon. This also follows from (19) and (20), which show that

$$
C^{*} \geqq \frac{1}{\pi} \int_{0}^{\pi} \frac{\sin u}{u} d u .
$$

Comparing this with (21) we see that

$$
C^{*}=\frac{1}{\pi} \int_{0}^{\pi} \frac{\sin u}{u} d u=.589490 \ldots .
$$

(ii) Let $U(\rho, \theta)$ be the Poisson integral of a function $F(t), 0 \leqq t<2 \pi$, of bounded variation and not constant. Thus

$$
U(\rho, \theta)=\frac{1}{2 \pi} \int_{-\pi}^{\pi} \frac{1-\rho^{2}}{1-2 \rho \cos (t-\theta)+\rho^{2}} F(t) d t .
$$

Since the total variation of $U(z)$ on the circle $|z|=\rho<1$ tends to the total variation of $F(t)$ over $(0,2 \pi)$, Theorem 1 gives

$$
\int_{D}\left|\frac{d}{d \rho} U_{n}(z)\right| d \rho \leqq \frac{2}{\pi} \int_{0}^{2 \pi}|d F(t)| .
$$

This, of course, may be obtained directly, by applying to the formula

$$
\frac{d}{d \rho} U_{n}(\rho, \theta)=\frac{1}{\pi} \int_{-\pi}^{\pi} \Delta_{n}(\rho, \theta-t) d F(t)
$$

the estimates for $\int_{-1}^{+1}\left|\Delta_{n}(\rho, t)\right| d \rho$. This direct approach shows that if, 
for simplicity, we take for $D$ the segment $(-1,+1)$, then the sign of equality occurs in (23) if and only if (a) $n=1$, (b) $F(t)$ is $C x(t)$, where $C$ is any constant, and $x(t)$ equals +1 inside $(-\pi / 2,+\pi / 2)$ and equals -1 inside the intervals $(-\pi,-\pi / 2)$ and $(\pi / 2, \pi)$. In other words, $U(\rho, \theta)$ must be

$$
C \sum_{\nu=1}^{\infty}(-1)^{\nu} \frac{\cos (2 \nu-1) \theta}{2 \nu-1} \rho^{2 \nu-1} .
$$

University of Pennsylvania 